\title{
Geolocalización de pacientes con cáncer bucal en la ciudad de Córdoba: factores de riesgo ambientales
}

\section{Geolocalization of patients with buccal cancer in the city of Córdoba: environmental risk factors}

\author{
Nieto AG, Unamuno V, Lazos J, Carrica A, Panico R, Brunotto M, Zarate AM \\ Universidad Nacional de Córdoba, Facultad de Odontología, Argentina.
}

\begin{abstract}
Pollution comes from activities that man performs at all levels. Many of the polluting substances do not have immediate action on health and cause long-term chronic diseases such as cancer. An important contribution in oral cancer research is to study the relationships between space and community health. The Geographic Information Systems constitute a computer set (software and hardware) that allows analyzing geographic and spatial data such as events, people and environmental characteristics. The objective of the study was to determine the spatial distribution of patients with oral cancer and other oral lesions, using Geographic Information Systems and establish possible association with the presence of different types of industries in the City of Córdoba. Methods:Retrospective study of clinical histories of patients of both sexes ( $n=476)$, who attended the External Clinic of the Chair of Stomatology "A" of the Faculty of Dentistry (UNC), period 2008 - 2014. The lesions were grouped into: benign, potentially malignant oral disorders (leucoplasias and oral lichens) and oral cancer. The patients were geolocated, by spatial localization of geographic coordinates, according to the address declared in the Clinical History. The industries were classified as Harmful and Dangerous. Planar type maps were elaborated with the program ArcGis 10.1, Esri. The statistics were performed using absolute and relative frequencies. The association between industrial zones and the presence of lesions was evaluated through Correspondence Analysis. Results: There was an association between the presence of non-malignant lesions with dangerous industries and malignant lesions with harmful industries. Areas with dangerous and harmful industries were associated with the presence of potentially malignant disorders. Discussion: Our results allowed us to determine environmental risk patterns in the city of Córdoba, which could be applied to analyze and / or solve different aspects of public health in relation to oral cancer.
\end{abstract}

KEYWORDS: Geographic information systems, harmful and dangerous industries, oral cancer

\section{Resumen}

La contaminación proviene de las actividades que realiza el hombre a todo nivel. Muchas de las sustancias contaminantes no tienen acción inmediata en la salud y originan enfermedades crónicas de larga duración como el cáncer.Un aporte importante en la investigación del cáncer oral es estudiar las relaciones entre el espacio y la salud comunitaria. Los Sistemas de Información Geográfica (SIG) constituyen un conjunto informático (de software y hardware) que permiten analizar datos geográficos y espaciales como eventos, personas y características ambientales.El objetivo de trabajo fue determinar la distribución espacial de pacientes con cáncer bucal y otras lesiones orales, utilizando Sistemas de Información Geográfica y establecer posible asociación con la presencia de industrias de diferentes tipos, en la Ciudad de Córdoba. Métodos: Estudio retrospectivo de Historias Clínicas de pacientes de ambos sexos (n=476), que asistieron al Consultorio Externo de la Cátedra "A" de Estomatología de la Facultad de Odontología (UNC), período 2008 - 2014. Las lesiones se agruparon en: benignas, desórdenes orales potencialmente malignos (leucoplasias y líquenes orales) y cáncer oral. Los pacientes se geolocalizaron, mediante localización espacial de coordenadas geográficas, según domicilio declarado en Historia Clínica.Las industrias fueron clasificadas en Nocivas y Peligrosas Se elaboraron mapas de tipo planar con programa ArcGis 10.1, Esri. La estadística se realizó mediante frecuencias absolutas y relativas. La asociación entre zonas industriales y presencia de lesiones se evaluó mediante Análisis de Correspondencia. Resultados: Se encontró asociación entre presencia de lesiones no malignas con industrias peligrosas y lesiones malignas con industrias nocivas. Las zonas con industrias peligrosas y nocivas se relacionaron con la presencia de desórdenes potencialmente maligno. Discusión. Nuestros resultados permitieron determinar patrones ambientales de riesgo en la ciudad de Córdoba, que podrían ser aplicados a analizar y/o solucionar diferentes aspectos de la salud pública en relación al cáncer bucal.

PALABRAS CLAVES: Sistemas de información geográfica, industrias nocivas y peligrosas, cáncer oral 


\section{Introducción}

La contaminación proviene de las actividades que realiza el hombre a todo nivel, es múltiple y se presenta en formas muy diversas, con asociaciones y sinergismos difíciles de prever ${ }^{1}$. Las sustancias contaminantes entran al organismo a través del agua, del aire y los alimentos, por inhalación, por contacto o por ingesta. Las fuentes de constituyentes químicos según la Organización Mundial de la Salud (OMS), son de ocurrencia natural o provienen de fuentes industriales y edificaciones humanas (industrias extractivas como minería, manufactura y procesos industriales, alcantarillado, residuos sólidos, fugas de gasolina), actividades agrícolas (abono, fertilizantes, pesticidas), tratamiento de agua o materiales en contacto con agua potable (coagulantes, subproductos de desinfección, tuberías de PVC, asbesto), pesticidas usados en agua para salud pública (plaguicidas usados en control de vectores), cianobacterias (lagos eutróficos), etc ${ }^{2}$. Entre los contaminantes químicos más peligrosos están: el asbesto, el benceno, el cloruro de vinilo, el carbón, el arsénico, los bifenilospoliclorados, el acetaldehído, el bromoformo, el cadmio, el cloroformo, el 1,2-dicloroetano, difenilhidrazina, el dinitrotolueno, el estireno, el formaldehido, el plomo y el tetracloruro de carbono. Estos contaminantes pueden ocasionar lesiones en el tracto respiratorio, cambios en la mucosa nasal y tráquea, irritación en los ojos, nariz y garganta, así como daños en el hígado, riñón, efectos inmunológicos $\mathrm{y}$ en el sistema nervioso central, conjuntivitis, dermatitis, lesiones en el sistema respiratorio y digestivo, malformaciones embrionarias, fatiga, jaquecas, mareos, depresión, anemia y por último muerte ${ }^{3-4}$. Muchas de estas sustancias no tienen acción inmediata en la salud de las personas y originan enfermedades crónicas de larga duración y generalmente de progreso lento, como enfermedades cardiacas, neurológicas, cáncer, diabetes y enfermedades crónicas respiratorias ${ }^{1}$.

El cáncer es considerado un problema de salud pública en todo el mundo. Según la Organización Mundial de la Salud (OMS), hay 10 millones de nuevos casos y más de 6 millones de muertes cada año por esta enfermedad en todo el mundo. Los cánceres localizados en la cavidad oral, definidos como cánceres de labio, lengua y boca (WHO, ICD 10 clasificación: C 01-06) representan el $48 \%$ de todos los cánceres de cabeza y cuello (HNC), y el $90 \%$ de estos son carcinomas de células ${ }^{5}$.

Un aporte importante en la investigación del cáncer oral es estudiar las relaciones entre el espacio y la salud comunitaria. Las herramientas de geo-procesamiento, y entre ellos los Sistemas de Información Geográfica (SIG) constituyen un conjunto informático (de software y hardware) que permiten introducir, almacenar, gerenciar, mostrar y analizar datos geográficos y espaciales o cualquier información que pueda estar relacionada con la localización geográfica , como eventos, personas y características ambientales ${ }^{6}$.Los SIG se destacan como una valiosa tecnología en la exploración de estas relaciones, contribuyendo así a una mejor comprensión entre el medio ambiente y la salud, con una rápida comprensión de los lugares donde se producen los problemas con mayor frecuencia; esto facilita el proceso continuo de planificación, seguimiento y evaluación de los servicios de salud ${ }^{7}$.Estudios recientes han mostrado la importancia del uso de los SIG como un método utilizado con cada vez más aceptación en estudios epidemiológicos, pues permiten proveer de importante información en el análisis de la distribución geográfica de las enfermedades, su relación con factores sociales, económicos y medioambientales, los agentes patogénicos como así también dilucidar los mecanismos de las enfermedades ${ }^{8}$.

Los SIG no son aún muy difundidos como herramienta en salud pública y mucho menos aún en lo referido a salud bucal. Pero su capacidad para integrar datos de los tres componentes epidemiológicos más importantes (persona, lugar y tiempo) los hace particularmente adecuados como una herramienta para la investigación epidemiológica del cáncer. Con respecto a la persona está bien establecido que muchos cánceres están relacionados con factores demográficos tales como raza y sexo. Utilizando los SIG la localización de los casos de cáncer pueden ser superpuestos con mapas de datos de la población para 
visualizar las relaciones entre los factores demográficos y los patrones del cáncer. Con respecto al lugar, los epidemiólogos han examinado tradicionalmente la variación geográfica en la incidencia del cáncer utilizando mapas ${ }^{9-10}$ La capacidad del SIG de manejar datos espaciales en una escala mucho más pequeña (al señalar la ubicación exacta de los casos de cáncer), junto con su capacidad para manejar múltiples niveles de la escala (grupo de bloque, área de censo, ciudad, condado, estado, etc.) aumentan la posibilidad de descubrir patrones espaciales, que no se cumplirían con los métodos epidemiológicos tradicionales. Además, la existencia de factores de riesgo ambientales conocidos para el cáncer, que pueden variar con la ubicación geográfica, se puede investigar con SIG. Con respecto al tercer factor tiempo, la información sobre la fecha del diagnóstico, la muerte o la recurrencia de los casos de cáncer puede ser ingresada en un SIG para poder examinar las relaciones temporales y espacio-temporales ${ }^{11}$.

En nuestra ciudad no existen estudios relacionados al uso de la epidemiología espacial en el cáncer bucal, por lo que resulta de gran importancia avanzar en la investigación de esta herramienta a fin de determinar patrones ambientales de riesgo en la ciudad de Córdoba para obtener datos relevantes que puedan ser aplicados a analizar y/o solucionar diferentes aspectos de la salud pública en relación al cáncer bucal. En esta ciudad existen muchas industrias situadas en zonas con alta concentración de habitantes. Las industrias pueden suponer un riesgo, no solo para los trabajadores, sino también para las personas que residen en su proximidad, ya que sus emisiones, que liberan sustancias tóxicas para el medio ambiente, son una fuente importante de contaminación del aire ${ }^{12}$.El objetivo de nuestro trabajo fue determinar la distribución espacial de pacientes con cáncer bucal y otras lesiones orales utilizando Sistemas de Información Geográfica (SIG) y establecer una posible asociación con la presencia de industrias de diferentes tipos, en la Ciudad de Córdoba, Córdoba, Argentina.

\section{Métodos}

Este trabajo se realizó en la Facultad de Odontología de la Universidad Nacional de Córdoba (Cátedras "A" de Biología Celular y Cátedra "A" de Estomatología) en convenio con el Observatorio Urbano Córdoba (OUC) de la Facultad de Filosofía y Humanidades, de la Universidad Nacional de Córdoba.

Se realizó un estudio retrospectivo a partir de Historias Clínicas de pacientes de ambos sexos $(n=476)$, que asistieron al Consultorio Externo de la Cátedra de Clínica Estomatológica "A" de la Facultad de Odontología de la Universidad Nacional de Córdoba, en el período comprendido entre los años 2008 y 2014.

Las edades de los pacientes estuvieron comprendidas entre 18 y 88 años. Los pacientes fueron clasificados en 3 grupos de edades, teniendo en cuenta la evolución natural del cáncer oral, entendida como una enfermedad crónica cuya incidencia va en aumento con la edad de la persona. Los grupos etarios fueron: a) pacientes menores de 45 años, b) entre 46 y 60 años y c) con más de 60 años ${ }^{13}$.

Con respecto a la residencia se consideró a los pacientes que tuvieran hasta 5 años de residencia en el domicilio actual dela Ciudad de Córdoba, los cuales fueron geolocalizados. Las lesiones de los pacientes se agruparon en: lesiones benignas (LB), desórdenes orales potencialmente malignos (DOPM) y cáncer oral (CO); las mismas habían sido confirmadas por diagnóstico histopatológico. Los DOPM fueron diagnosticados como leucoplasias y líquenes orales (WHO classification ICD-10 K00-K14, 2010) ${ }^{14}$.

Las industrias consideradas fueron clasificadas en: a) Nocivas: las que por su tipo, tecnología, procesos y/o escalas o magnitudes, producen daños, perjuicios y perturbaciones $\mathrm{o}$ efectos tóxicos $\mathrm{o}$ perniciosos sobre el entorno y b) Peligrosas, inflamables o explosivas: las que por su tipo, tecnología, procesos y/o escalas o magnitudes, producen situaciones de riesgo o inseguridad inminente sobre el entorno por explosiones, combustibilidad, inflamabilidad o toxicidad. Esta clasificación es la establecida por la Ordenanza $\mathrm{N}^{\circ} 8133$ sobre el Uso del Suelo del Honorable Concejo Deliberante de la Ciudad de Córdoba. 
Se elaboraron mapas de tipo planar con el programa ArcGis 10.1, Esri. Las capas geográficas que se utilizaron fueron son: "manzanas.shp" e "industrias.shp" de la Ciudad de Córdoba (2012) brindadas por el OUC.

Las variables estudiadas fueron: sexo, edad, lesiones bucales, residencia actual, hábitos e industrias en zonas de influencia al domicilio.

\section{Análisis Estadístico}

Las variables que se estudiaron fueron: lesiones bucales, sexo, edad, residencia actual, tipo de industrias.

La descripción estadística de las mismas se realizó mediante frecuencias absolutas y relativas. La asociación entre las zonas industriales y la presencia de lesiones se evaluó mediante método multivariado exploratorio de Análisis de Correspondencia, que permitió representar gráficamente filas y columnas de tablas de contingencia múltiples.

\section{Resultados}

Del total de pacientes que asistieron a la consulta, el $61 \%$ fue de sexo femenino.

Las lesiones malignas y potencialmente malignas fueron encontradas en mayor número en pacientes de entre 56-88 años.

Las lesiones más prevalentes fueron las lesiones benignas (59\%), seguidas por DOPM y finalmente, los tumores malignos.

Considerando solamente las lesiones premalignas y malignas se observó que el tipo más significativamente prevalente $(\mathrm{p}=0,0001)$ fueron los DOPM, siendo el género masculino el más afectado.

Las lesiones orales en relación a hábitos de riesgo, no se observaron diferencias significativas $(p>0,05)$ entre los pacientes que fumaban y/o bebían alcohol y aquellos que no lo hacían, y el desarrollo de las mismas (Fig. 1).

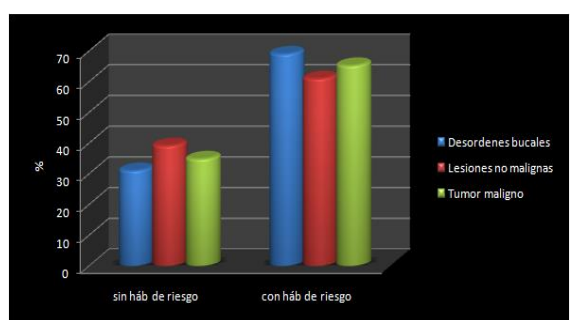

Figura 1. Porcentaje de pacientes con hábitos de riesgo en relación al desarrollo de lesiones bucales.

La geolocalización de los pacientes y de las industrias se muestra en la Fig. 2.

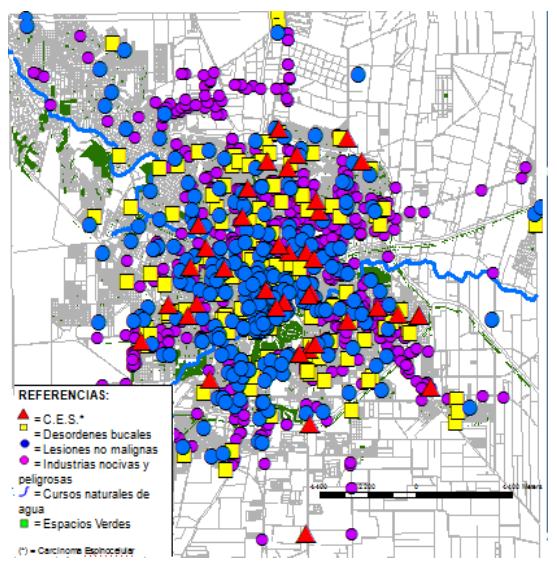

Figura 2. Geolocalización de pacientes con lesiones orales y de industrias peligrosas y nocivas, en la Ciudad de Córdoba, Córdoba, Argentina

La distribución de los pacientes con lesiones potencialmente malignas se muestra en la Fig. 3 y la distribución de los pacientes con cáncer oral se muestra en la Fig. 4.



Figura 3.Distribución de lesiones potencialmente malignas (o premalignas) en la ciudad de Córdoba (periodo estudiado 2008-2014.) 


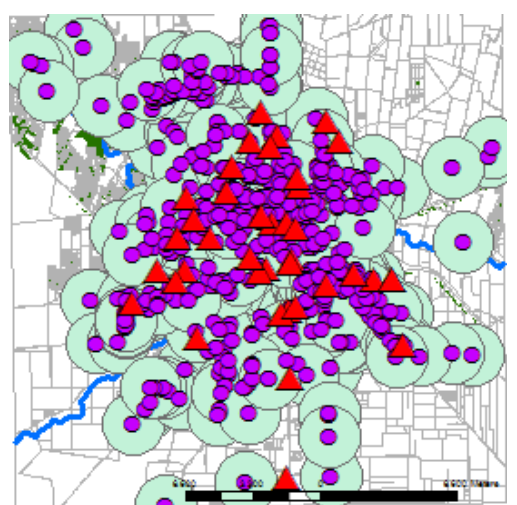

Figura 4. Distribución de lesiones orales malignas (o cáncer oral) en la ciudad de Córdoba (periodo estudiado 2008-2014).

El análisis multivariado de correspondencia fue realizado con los dos primeros ejes (inercia acumulada en primer eje $87,8 \%$ primer eje, en el segundo eje 12,2\%). Se encontró asociación entre la presencia de lesiones no malignas con industrias peligrosas y lesiones malignas con industrias nocivas. Las zonas con industrias peligrosas y nocivas se relacionaron con la presencia de desórdenes potencialmente malignos (Fig. 5).

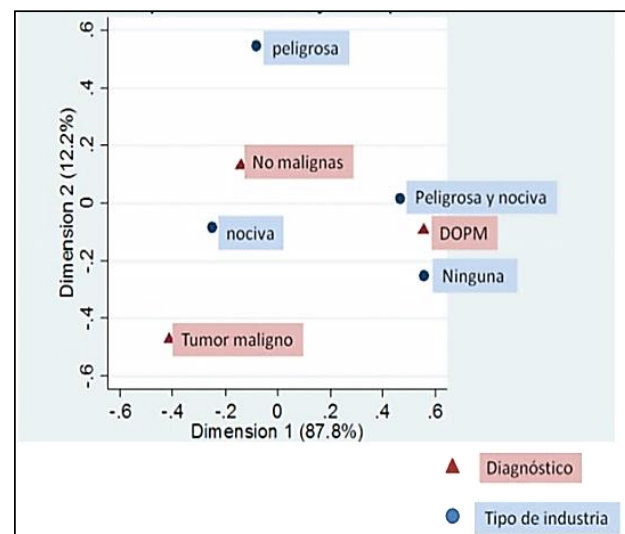

Figura 5. Análisis multivariado de correspondencia. El análisis multivariado de correspondencia fue realizado con los dos primeros ejes (inercia acumulada en primer eje $87,8 \%$ primer eje, en el segundo eje $12,2 \%)$.

\section{Discusión}

La utilización de herramientas como las que brindan los sistemas de información geográfica (SIG), han permitido a los investigadores explorar nuevas relaciones en la distribución del cáncer que no habían sido determinadas con métodos epidemiológicos tradicionales ${ }^{15}$. $\mathrm{La}$ capacidad de los sistemas de información geográficos para cuantificar con exactitud los datos sobre la exposición medioambiental, ha llevado a poner más énfasis en los factores ambientales en lo que a investigación del cáncer oral se refiere. La superposición de puntos y polígonos y la proximidad mediante buffer son técnicas SIG especialmente aplicables a la visualización de la relación entre la exposición ambiental y el cáncer. Esto debido a que el investigador puede superponer la distribución o los casos y / o controles (representados por puntos) con la distribución de las características ambientales (representadas por polígonos) para generar hipótesis sobre los factores de riesgo, que luego se pueden estudiar con los diseños de estudios epidemiológicos tradicionales, tales como cohorte o casocontrol ${ }^{16}$.

Tanser et al, $2002^{17}$ en una revisión de literatura sobre salud en África, reveló que los SIG adquieren gran importancia en las denominadas enfermedades «medioambientales». En ciertas enfermedades como las transmitidas por vectores (por ejemplo, malaria, esquistosomiasis, infecciones por helmintos humanos y tripanosomiasis) el componente ambiental es determinante así como la intensidad de sus factores.

Por otra parte es importante conocer y aplicar las GIS para crear nuevas bases de datos para calcular el grado de exposición ambiental a los carcinógenos; Lewis-Michl et $a l, 1996^{18}$, en un estudio caso control realizado sobre la relación entre la exposición a contaminantes químicos y el cáncer de mama utilizando el historial de ubicación de casos de cáncer de mama e instalaciones de fábricas durante un período de veinte años, considerando la distancia de residencia de las personas y las fuentes de contaminantes químicos tóxicos, encontraron una asociación significativa entre las variables estudiadas estableciendo así una base de datos importante para el estudio de la enfermedad dejando establecido el efecto de las industrias relacionadas a los ambientes donde vivían estas personas ${ }^{15}$.

En nuestra ciudad no existen investigaciones relacionados al uso de la 
epidemiología espacial en el cáncer bucal, por lo que es el primer estudio de geolocalización de DOPM y cáncer oral en relación a características ambientales de una comunidad subyacente en Argentina.

En este trabajo se encontró una asociación significativa entre el desarrollo del cáncer oral y la presencia de industrias nocivas. Se conoce que el cáncer se desarrolla a partir de la acumulación y selección sucesiva de alteraciones genéticas y epigenéticas, que permiten a las células sobrevivir, replicarse $\mathrm{y}$ evadir mecanismos reguladores de apoptosis, proliferación y del ciclo celular; también los mecanismos responsables de mantener y reparar el DNA se encuentran modificados 19. El cáncer es una enfermedad de etiología multifactorial que en el $80 \%$ de los casos se debe a factores exógenos o externos como, entre otros, los contaminantes ambientales, el estilo de vida el consumo de alcohol en exceso y el hábito de fumar, y en un $20 \%$ a factores endógenos, genéticos y epigenéticos ${ }^{14}$.

Con respecto a los contaminantes ambientales las anormalidades producidas en el material genético de las células pueden ser provocadas, entre otras causas, por distintos agentes carcinógenos, como la radiación ionizante, ultravioleta, productos químicos procedentes de las industrias, y de la contaminación en general.

White y Aldrich, $1999^{20}$, en un estudio sobre cáncer pediátrico, definieron zonas basadas en buffers de sitios de desechos peligrosos, debido a su interés en la proximidad a las toxinas ambientales como un factor de riesgo para cáncer infantil. Mediante la definición de zonas según diferentes tipos de exposición ambiental, determinaron diferentes hipótesis sobre factores de riesgo ambientales.

El hábito de fumar y la ingestión de alcohol son dos factores de alto riesgo de padecer cáncer bucal. Alrededor del 80 al $85 \%$ de los pacientes con cáncer bucal tienen una importante historia de alcohol-tabaco. En enfermedades crónicas no transmisibles, como el cáncer bucal, habitualmente los hábitos de riesgo de fumar y beber aportan un porcentaje acumulado de aproximadamente $43 \%$, mientras que el medio ambiente aporta una carga patogénica del $19 \%{ }^{21}$. En nuestro trabajo no se encontraron diferencias significativas entre pacientes con/sin estos hábitos de riesgo en cuanto al hábito de fumar pero si encontramos asociación entre las industrias peligrosas y nocivas (principalmente las correspondientes al sector eléctrico, químico, de plásticos y plaguicidas) con la presencia de DOPM, por lo tanto consideramos que la influencia de las industrias es importante en el avance de las lesiones precancerizables hacia la malignidad; la exposición a las sustancias producidas por estas industrias podrían constituir un factor determinante también para la presencia de los mismos ya que los DOPM constituyen un grupo de patologías complejas en las cuales el factor tiempo es relevante lo que podría estar asociado al efecto contaminante sostenido, característico de las industrias nocivas.

Las investigaciones realizadas para el control del cáncer tratan de reducir la carga del mismo estudiando poblaciones definidas. El primer paso en el control de esta enfermedad es identificar donde la incidencia de cáncer es elevada, para sugerir así intervenciones con ubicaciones específicas. Es por ello que los SIG permiten proveer soluciones, jugando así un rol importante en el monitoreo del cáncer.

Entendiendo que los datos se muestran geográficamente utilizando un SIG de gestión, es conveniente establecer colaboraciones con instituciones de investigación para probar hipótesis y distribuciones de enfermedades modelo ${ }^{17}$. Del mismo modo, las aplicaciones de investigación informarán a las aplicaciones de gestión de SIG para planificar la asignación óptima de recursos y estrategias de intervención. En nuestra ciudad no existen estudios relacionados al uso de la epidemiología espacial en el cáncer bucal, por lo que resulta de gran importancia avanzar en la investigación de esta herramienta a fin de determinar patrones ambientales de riesgo en la ciudad de Córdoba, para obtener datos relevantes que puedan ser aplicados a analizar y/o solucionar diferentes aspectos de la salud pública en relación al cáncer bucal.

Todos los autores declaran que no existen conflictos potenciales de interés con respecto a la autoría y/o publicación de este artículo. 
All authors declare that there are no potential conflicts of interest with respect to the authorship and / or publication of this article

\section{Referencias}

1. Chung B. Control de los contaminantes químicos en el Perú. Rev. perú. med. exp. salud pública [Internet]. 2008; 25( 4 ): 413-418. Disponible en:

2. de Rosa CT, El-Masri HA, Pohl H, Cibulas W, Mumtaz MM. Implications of chemical mixtures in public health practice. $J$ ToxicolEnvironHealth B Crit Rev. 2004; 7(5): 339-50.

3. Karr CJ, Solomon GM, Brock-Utne AC. Health effects of common home, lawn, and garden pesticides. PediatrClin North Am. 2007; 54(1): 63-80.

4. Avila J, Honorio L, Chira C, Samatelo H, Urbano C. Intoxicación aguda por inhalación de acrilato de etilo, Lima 2002. Rev Peru Med ExpSaludPublica. 2005; 22(4): 267-73.

5. Gonzalez Segura I, Secch D, Carrica A, Barello R, Arbelo D, Burgos A, Brunotto M, Zarate A. Exfoliative cytology as a tool for monitoring pre-malignantand malignant lesions based on combined stains andmorphometry techniques. J Oral Pathol Med. 2015 Mar; 44(3):178-84.

6. Perera I, Kruger E, Tennant M. 2010. Rural Public Dental Clinic Distribution in Three States of Australia: Using Spatial Analysis to Inform Management and Planning of Services. Asia PacificJournal of Health Management; 5(1): 4046.

7. JacquezG,Greiling D. Geographic boundaries in breast, lung and colorectal cancers inrelation to exposure to air toxics in Long Island, New York International Journal of Health Geographics 2003, 2:1-22

8. PereiraS, Ambrosano G , Cortellazzi K , Tagliaferro M ,Vettorazzi C, Ferraz S , Meneghim M, Pereira A. Geographic Information Systems (GIS) in Assessing Dental Health. Int. J. Environ. Res. Public Health 2010, 7, 2423-2436.

9. Pickle Lw, Szczur M, Lewis Dr, Stinchcomb Dg. The crossroads of GIS and health information: a workshop on developing a research agenda to improve cancer control. Int $\mathbf{J}$ Health Geogr. 2006; 5:51.

10. Plesko I, Obsitnikova A, Kramarova E. Mapping in epidemiological studies and control of cancer:
Experiences from Slovakia. J Environ PatholToxicolOncol. 1996; 15:143-7

11. Najafabadi, A. T.Applications of GIS in Health Sciences. Shiraz E-Med J. 2009; 10:221-30.

12. Zayas Mujica R, Cabrera Cárdenas Ul. Los tóxicos ambientales y su impacto en la salud de los niños. Rev Cubana Pediatr. 2007; 79( 2 ):2025.

13. Dericia J, Gonzalez Segura I, Brezzo M, Croharé L, Secchi D, Malberti A, Zarate A.Estudio citomorfométrico de pacientes con cáncer y desórdenes potencialmente malignos de la cavidad bucal. Acta odontológica venezolana 2012; 50(3)

14. Zarate A, Don J, Secchi D, Carrica a, Galindez Costa F, Panico R, Brusa M, Barra J, Brunotto M. Study of the tp53 codon 72 polymorphism in oral cancer and oral potentially malignant disorders in argentinean patients. Tumor Biology.2017; 39(5):1010428317699113ISSN: 1010-4283 (Print), 1423-0380 (Electronic)..

15. Najafabadi At, M Pourhassan M Integrating the geographic information system into cancer research. Indian J Cancer. 2011, 48(1):105-109.

16. Yifei S. Determining the size of spatial clusters in focused tests: Comparing two methods by means of simulation in a GIS* J GeographSyst (2002) 4:359-370

17. Tanser F, Sueur D The application of geographical information systems to important public health problems in Africa. International Journal of Health Geographics 2002 1:1-12

18. Lewis-Michl EL, Melius JM, Kallenbach LR, Ju CL, Talbot TO, Orr MF, et al. Breast cancer risk and residence near industry or traffic in Nassau and Suffolk Counties, Long Island, New York. Arch Environ Health 1996; 51:255-65.

19. Kumaraswamy KL, Vidhya M. Human papilloma virus and oral infections: an update. $\mathrm{J}$ Cancer Res Ther 2011; 7:120-7

20. White E, Aldrich TE. Geographic studies of pediatric cancer near hazardous waste sites. Arch Enviro Health 1999; 54:390-7.

21. Martinez L E, Quiroz C, Daniels C F, Montoya E A. Contaminación atmosférica y efectos sobre la salud de la población, Medellín y su área metropolitana. 2007 Disponible en: http://www.metropol.gov.co/aire/contenidos.php ?seccion $=1$.

Correspondencia a / Corresponding to:

Dra. Ana María Zarate

Facultad de Odontología UNC-Córdoba Argentina.

Correo electrónico (e-mail): azgelfo@hotmail.com 